\section{Cureus}

Received 12/12/2018

Review began 01/25/2019

Review ended 03/20/2019

Published 04/03/2019

\section{(c) Copyright 2019}

Bishop et al. This is an open access article distributed under the terms of the Creative Commons Attribution License CC-BY 3.0., which permits unrestricted use, distribution, and reproduction in any medium, provided the original author and source are credited.

\title{
Development of a Three-dimensional Printed Emergent Burr Hole and Craniotomy Simulator
}

\author{
Nicole Bishop ${ }^{1}$, Darrell Boone ${ }^{2}$, Kerry-Lynn Williams ${ }^{1}$, Roger Avery ${ }^{3}$, Adam Dubrowski ${ }^{4}$ \\ 1. Medical Education and Simulation, Memorial University of Newfoundland, St. John's, CAN 2. General \\ Surgery, Memorial University of Newfoundland, St. John's, CAN 3. Neurosurgery, Memorial University of \\ Newfoundland, St. John's, CAN 4. Emergency Medicine, Memorial University of Newfoundland, St. John's, \\ CAN
}

$\square$ Corresponding author: Nicole Bishop, n.bishop@mun.ca

Disclosures can be found in Additional Information at the end of the article

\section{Abstract}

Patients with a traumatic head injury (THI) require immediate surgical intervention, as rapidly expanding intracranial hematomas can be imminently life-threatening, not permitting transfer time to neurosurgical care in a tertiary care center. In rural and remote areas, where neurosurgeons may not be readily available, surgical intervention by Community General Surgeons (CGS) may be required. Currently, the CGS in Newfoundland and Labrador (NL) do not have access to, or have experience training with, an emergent burr hole/craniotomy (EBHC) simulator. One of the barriers is the availability of inexpensive and reliable simulators to practice these skills. Therefore, a low-cost, three-dimensional (3D) printed EBHC simulator was designed and 3D-printed by MUN Med 3D (St John's, NL).

The aim of this technical report is to assess the need for such simulator in rural and remote healthcare centers and report on the iterative development of the EBHC simulator. The 3Dprinted EBHC simulator developed by MUN Med 3D was utilized during a general surgery workshop at the $26^{\text {th }}$ Annual Rural and Remote Medicine Conference in St. John's, NL. A total of six 3D-printed EBHC simulators were provided for the hour and a half workshop. At the end of the workshop, 16 participants were asked to provide feedback on the need for this simulator in their rural or remote environment as well as feedback on the physical attributes. The feedback received from the participants was overall positive, informative, and supported the need for the 3D-printed EBHC simulator.

Categories: Medical Education, General Surgery, Quality Improvement Keywords: rural healthcare, three-dimensional printing, craniotomy, simulation based medical education

\section{Introduction}

Patients with a traumatic head injury (THI), such as an expanding epidural hematoma (EDH) or subdural hematoma (SDH), require urgent operative intervention to relieve pressure on the brain and control hemorrhaging [1]. In rural and remote areas, where neurosurgeons may not be readily available, surgical intervention by Community General Surgeons (CGS) may be required [2-4]. For a THI, immediate surgical intervention is essential, as rapidly expanding intracranial hematomas can be imminently life-threatening, not permitting transfer time to neurosurgical care in a tertiary care center [1]. In a case where a patient presents with an EDH or SDH, a CGS is confronted with a difficult decision: operate, in undesirable circumstances in 
teleconsultation with a neurosurgeon, or transfer the patient to a tertiary care center with the potential for adverse consequences due to delay of care.

While evidence shows that the early decompression and hematoma evacuation of an EDH or SDH improves patient outcomes [1,5-9], Donovan et al. [8] suggest that it is best for this emergency procedure is treated by experienced neurosurgeons in a medical facility equipped with a computed tomography (CT) scanner. In addition, Rinker et al. [1] note that an inexperienced surgeon may encounter difficulties with the emergency burr hole craniotomy and that well-intentioned efforts may actually delay transfer to an experienced neurosurgeon.

However, this optimal surgical environment is not readily available in several rural hospitals, as they may not have access to CT scanners and/or have a neurosurgical presence. Therefore, the next best scenario is for a trained-to-proficiency CGS to assess the emergency situation in teleconsultation with a neurosurgeon. If surgical intervention is deemed appropriate due to time-sensitivity, the trained-to-proficiency CGS, remotely assisted by a neurosurgeon, may perform the procedure in a timely matter to prevent progressive neurological impairment or possible death of the patient [1] followed by an emergent transfer to a facility with a neurosurgeon.

Since there are currently a small number of clinical encounters for CGSs to develop skills related to decompression and hematoma evacuation of an EDH or SDH (personal communication with Dr. Darrell Boone and Dr. Michael Parsons), simulation may provide a good training platform to augment the transition from an inexperienced to a trained-toproficiency CGS. To date, simulation has become an excellent addition to healthcare education, as it promotes skill acquisition and maintenance through hands-on experience [1]. Simulation has been shown to increase the competency and confidence of individuals through the rehearsal of high acuity, low occurrence (HALO) procedural skills, resulting in a reduction of errors [10]. However, currently, the CGSs in rural and remote areas of Newfoundland and Labrador (NL) do not have access to or have experience training with, an emergent burr hole/craniotomy (EBHC) simulator (personal communication with Dr. Darrell Boone and Dr. Michael Parsons). Therefore, the first time they are likely to encounter this situation will be in the emergency setting, highlighting the fact that the CGS need to be skilled but, in most cases, lack the opportunity to develop and maintain these skills.

Subsequently, a three-dimensional (3D)-printed EBHC simulator was designed and printed with the purpose of being incorporated into a simulation-based medical education (SBME) curriculum developed collaboratively by neurosurgeons and CGSs, specifically delivered in rural and remote areas. In this conception, the EBHC simulator is one of the components of a simulation curriculum to train procedural skills related to EBHC. Therefore, the main purpose of this technical report is to assess the need for such simulators in rural and remote areas and report on the iterative development of the EBHC simulator.

\section{Technical Report}

\section{Context}

In NL, the population $(525,355$ as of July 1,2018$)$ [11] is dispersed over a large geographical area $(405,720$ square $\mathrm{km})$ [12], with only one tertiary hospital specializing in neurosurgery - the Health Science Centre (St. John's) [13]. This widely dispersed population leads to long transport times (Table 1) [14-15] that may have potentially detrimental effects on those who have THI, which requires timely, operative intervention. 


\section{Cureus}

\section{Location}

Happy Valley-Goose Bay (HVGB), Labrador

St. Anthony, Newfoundland

Port aux Basques, Newfoundland

\section{Average Transfer Time to Tertiary Care Center}

10 min drive to HVGB airport $+2 \mathrm{hr} 20$ min flight +10 min drive to HSC 45 min drive to St. Anthony airport $+1 \mathrm{hr} 10 \mathrm{~min}$ flight+ 10 min drive to HSC

$2 \mathrm{hr}$ drive to Stephenville airport $+1 \mathrm{hr}$ flight+ 10 min drive to HSC

\section{TABLE 1: Average transfer time from rural communities to a tertiary care center in NL}

HSC - Health Sciences Centre, the tertiary care center in St. John's, Newfoundland

NL: Newfoundland and Labrador

\section{Inputs}

The design time for the simulator (second iteration) was approximately 20 hours with a direct cost of approximately $\$ 396.60$ (not including overheads). Assembly time for each unit was 40 minutes, with an associated direct cost of $\$ 11.90$. Note that the design costs are based on a digital modeler time of $\$ 19.83$ /hour and assembly time on a mid-level pay scale for co-op students of $\$ 19.83 /$ hour. The total cost of the EBHC simulator, including design time and the materials mention in Table 2 was $\$ 440.02$. However, this is the cost for the first designed simulator; additional units of the simulator would be costed at materials and assembly only, totaling $\$ 44.02$.

\begin{tabular}{|c|c|c|c|c|}
\hline Simulator Component & Material & $\begin{array}{l}\text { Layer } \\
\text { Height }\end{array}$ & Infill & Cost \\
\hline Base & ABS & $0.20 \mathrm{~mm}$ & $25 \%$ & $\$ 5.01$ \\
\hline Clamps for base & ABS & $0.20 \mathrm{~mm}$ & $25 \%$ & $\$ 1.23$ \\
\hline Brain & TPU (Ninjaflex) & $0.15 \mathrm{~mm}$ & $25 \%$ & $\$ 6.60$ \\
\hline Skull & ABS & $0.20 \mathrm{~mm}$ & $25 \%$ & $\$ 4.47$ \\
\hline Dural membrane & Latex (latex gloves) & $N / A$ & $\mathrm{~N} / \mathrm{A}$ & $\$ 1.50$ \\
\hline Blood clots & Silicone Gel & $\mathrm{N} / \mathrm{A}$ & $\mathrm{N} / \mathrm{A}$ & $\$ 0.25$ \\
\hline Skin & Silicone & N/A & N/A & $\$ 7.00$ \\
\hline \multirow[t]{2}{*}{$\begin{array}{l}\text { Additional hardware to secure the simulator } \\
\text { together }\end{array}$} & $\begin{array}{l}1 / 4 "-20 \text { x 1.5" bolts, 1/4" washers, 1/4" nuts, } \\
\text { Epoxy }\end{array}$ & N/A & $\mathrm{N} / \mathrm{A}$ & $\$ 6.06$ \\
\hline & & & Total & $\$ 32.12$ \\
\hline
\end{tabular}

TABLE 2: Breakdown of the simulator materials, print time, and cost per component 


\section{Cureus}

\section{Process}

Simulator

The assembly of the 3D-printed EBHC simulators was divided into two categories: SDHs (accumulation of blood between the brain and the dura) [16] and three EDHs (accumulation of blood between the dura and the skull) [17]. Each individual simulator took approximately 40 minutes to construct; from individual printed components to finished product. To construct the EBHC simulator, the brain was inserted into the simulator base (Figure 1a), then depending on epidural or subdural models, the blood clots were placed. For the SDH simulator model, the blood clots were added before the placement of the dura membrane. For the EDH simulator model, the dura membrane was placed on top of the brain, followed by the blood clots. The skull was then placed. The dura membrane and skull were secured to the base using epoxy. Finally, the skin was draped over the skull (Figure $1 b$ ) and secured using the clamps on the base and additional hardware.

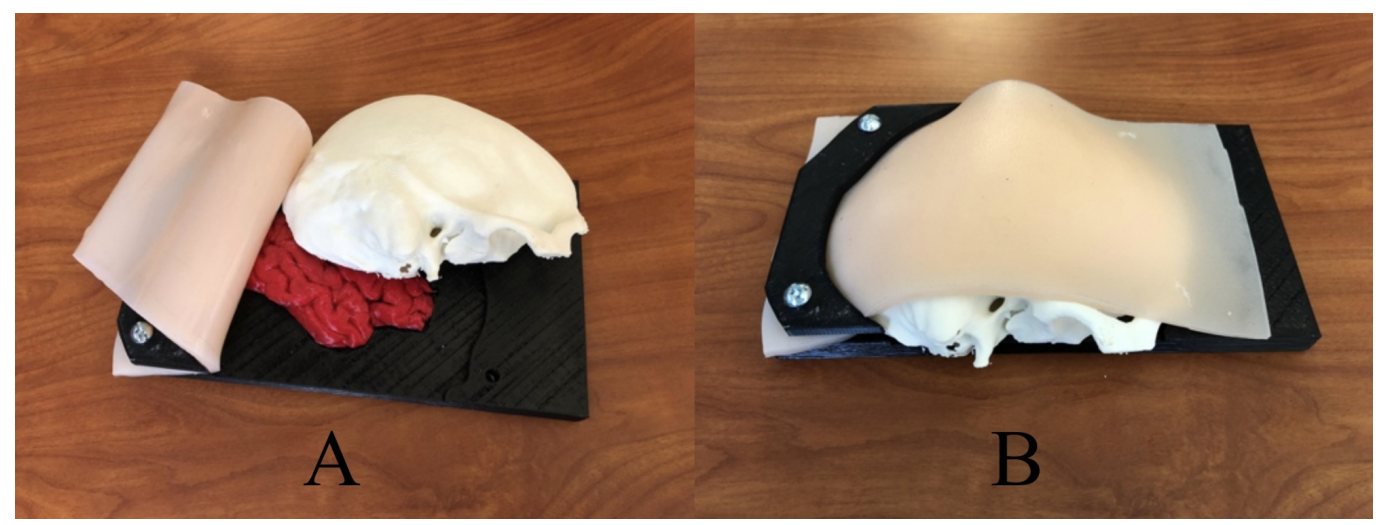

FIGURE 1: Construction stages of an emergent burr hole/craniotomy simulator

A - The first stage of the simulator construction (base, brain, skull, and skin)

B - The final stage of the simulation construction (the skin was draped over the skull and secured using the clamps on the base and additional hardware)

Need Assessment and Feedback

A needs assessment of the simulator for rural and remote training and feedback on the physical attributes of the 3D-printed EBHC simulator were collected after a 1.5 hour, hands-on workshop at the 26th Annual Rural and Remote Medicine Conference in St. John's, NL. This conference, hosted by the Society of Rural Physicians of Canada, targeted healthcare professionals who are currently practicing, or those who look to practice, in rural and remote areas of Canada. A total of 16 individuals attended the workshop. All individuals indicated that they were rural general practitioners (GP), with two individuals indicating they additionally completed enhanced surgical skills. This sample was used as a convince sample. A local general surgeon (Dr. Darrell Boone) referred to as the educator, led the workshop. The educator began the workshop with a 20-minute PowerPoint presentation describing the emergency burr hole and craniotomy procedures followed by the background on why and how the first iteration of the EBHC simulator was developed (Appendix A). The presentation also informed the participants of the variable transport times from rural areas to tertiary centers within Canada and the implications of these times. After the presentation, an informal 15-minute 


\section{Cureus}

demonstration on the EBHC simulator was provided by the educator (Figure 2).

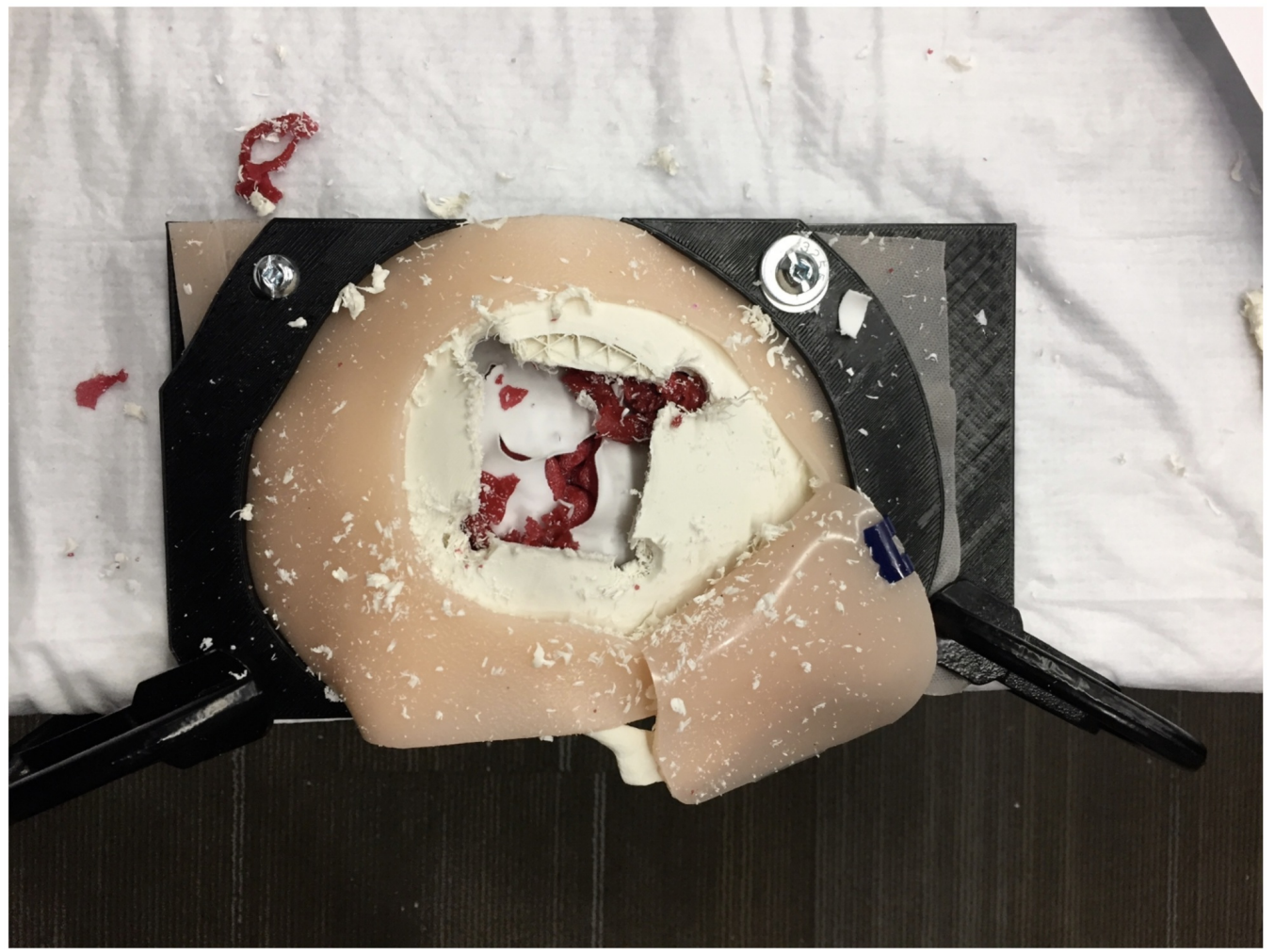

\section{FIGURE 2: Emergent burr hole/craniotomy simulator after a 15- minute demonstration by an educator}

Six 3D-printed EBHC simulators were set up on two standard tables, three simulators per table. The participants were given 45 minutes to "play" with the EBHC simulators and were asked to evaluate the realism of the physical attributes of the EBHC simulator (i.e. bone, skin) on the simulator evaluation survey. They were unable to assess the EBHC simulator as an educational tool, as they were not content experts (neurosurgeons and experienced general surgeons) in the specific procedural skills demonstrated.

\section{Outcomes}

Nine participants, out of the 15 who attended the workshop completed this survey. The survey posed open-ended questions to receive feedback concerning the need for an EBCH simulator in their practice setting and recommended improvements for future iterations of the physical attributes of the 3D-printed EBHC simulator. It was noted that while THI emergency situations are rare, they can and do occur and multiple participants indicated that they feel simulation training is becoming critical to learning and that they see a need for this skill in their rural and remote environment. Additionally, to the knowledge of the participants, there are no easily reproducible (3D-printed), low-cost simulators they are aware of to practice these procedural skills. Through informal feedback, one participant indicated that the CGS in their rural hospital encountered this emergency situation and that the CGS had no prior practice or experience in the procedures.

Pertaining to the physical attributes of the EBHC simulator, participants indicated that there was a need for blood to be incorporated into the 3D-printed EBCH simulator, and one 
respondent also provided ideas on how to better secure the skull to the base using gaskets. Furthermore, participants indicated that the texture and feel of the bone were realistic but the skin was too thick to be realistic. The educator also provided additional comments on how to improve the physical attributes of the EBHC simulator. He commented on the fact that the size of the brain was not anatomically correct for the size of the skull that was being used. As well, the colors of the dura membrane and brain were not accurate.

\section{Discussion}

The feedback received from the participants was overall positive, informative, and supported the need for a 3D-printed EBHC simulator in simulation curriculum for rural and remote locations. The implementation of the EBHC simulator into simulation training should focus on systematically addressing who the appropriate learners should be, where the liabilities are, and the relative weight of possible positive outcomes vs complications. With CGSs undergoing appropriate training, the concerns of Rinker et al. (1998) of inexperienced surgeons encountering difficulties with performing emergency burr holes and craniotomies may be addressed [1].

The recommendations provided to MUN Med 3D were included in the next iteration of the EBHC simulator (Figure 3). Once the revisions are complete, the task trainer is scheduled to undergo a comprehensive test of face and content validity by experienced content experts (neurosurgeons and experienced general surgeons) to ensure that the task trainer is anatomically correct and provides an overall realistic experience.
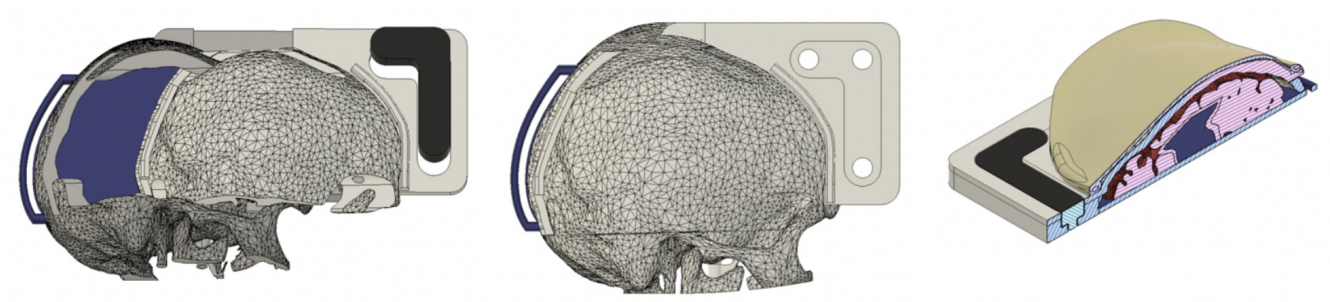

FIGURE 3: Iterative design after analyzing feedback

\section{Conclusions}

To the author's knowledge, there are currently no EBHC bench-top simulators on the market and no comparable simulator is incorporated into the current medical curriculum in NL. The current report shows that there is a need for the simulator and that after systematic validation of the 3D-printed EBHC simulator, it has the promise to be a cost-effective addition to an SBME curriculum. Currently, the cost of similar commercially available high-fidelity simulators are magnitudes more expansive, making them potentially prohibitive outside large, well-funded neurosurgical training programs. The developed simulator may fill the identified educational gap and potentially remove the high-risk and high-stress environment of performing these procedural skills for the first time in a real-life situation.

\section{Additional Information \\ Disclosures}

Human subjects: Consent was obtained by all participants in this study. Animal subjects: All authors have confirmed that this study did not involve animal subjects or tissue. Conflicts of 
interest: In compliance with the ICMJE uniform disclosure form, all authors declare the following: Payment/services info: All authors have declared that no financial support was received from any organization for the submitted work. Financial relationships: All authors have declared that they have no financial relationships at present or within the previous three years with any organizations that might have an interest in the submitted work. Other relationships: All authors have declared that there are no other relationships or activities that could appear to have influenced the submitted work.

\section{Acknowledgements}

The authors would like to acknowledge the hard work of Nora Boone, Michael Bartella,s and Stephen Ryan. In conjunction with Dr. Boone and Dr. Avery, Nora, Michael, and Steven developed the first iteration of the emergent burr hole and craniotomy simulator. Their work was recognized at the Youth Can Innovate Awards in Regina, Saskatchewan, Canada (2017), where Nora won first-place at the nation-wide science fair.

\section{References}

1. Rinker CF, McMurry FG, Groeneweg VR, Bahnson FF, Banks KL, Gannon DM: Emergency craniotomy in a rural level III trauma center. Trauma Surg Acute Care. 1998, 44:984-990.

2. Eaton J, Hanif AB, Mulima G, Kajombo C, Charles A: Outcomes following exploratory burr holes for traumatic brain injury in a resource poor setting. World Neurosurg. 2017, 105:257264. 10.1016/j.wneu.2017.05.153

3. Rosenfeld JV: Who will perform emergency neurosurgery in remote locations? . ANZ J Surg. 2015, 85:600. 10.1111/ans.13207

4. Treacy PJ, Reilly P, Brophy B: Emergency neurosurgery by general surgeons at a remote major hospital. ANZ J Surg. 2005, 75:852-857. 10.1111/j.1445-2197.2005.03549.x

5. Stone JL, Lowe RJ, Jonasson O, et al.: Acute subdural hematoma: direct admission to a trauma center yields improved results. Trauma Surg Acute Care. 1986, 26:445-450. 10.1097/00005373-198605000-00006

6. Seelig JM, Becker DP, Miller JD, Greenberg RP, Ward JD, Choi SC: Traumatic acute subdural hematoma: major mortality reduction in comatose patients treated within four hours. N Engl J Med. 1981, 304:1511-1518. 10.1056/NEJM198106183042503

7. Becker DP, Miller JD, Ward JD, Greenberg RP, Young HF, Sakalas R: The outcome from severe head injury with early diagnosis and intensive management. J Neurosurg. 1977, 47:491-502. 10.3171/jns.1977.47.4.0491

8. Donovan DJ, Moquin RR, Ecklund JM: Cranial burr holes and emergency craniotomy: review of indications and technique. Mil Med. 2006, 171:12-19.

9. Nehls DG, Mendelow DA, Graham DI, Teasdale GM: Experimental intracerebral hemorrhage: early removal of a spontaneous mass lesion improves late outcome. Neurosurgery. 1990, 27:674-682.

10. Newfoundland and Labrador Statistics Agency . (2018). Accessed: July 1, 2018: https://www.stats.gov.nl.ca.

11. About Newfoundland and Labrador. Land area . (2018). Accessed: July 1, 2018: https://www.gov.nl.ca/aboutnl/area.html.

12. The College of Physicians and Surgeons of Newfoundland and Labrador . (2018). Accessed: July 1, 2018: https://www.cpsnl.ca/WEB/CPSNL/Physician_Search_-_Specialists.aspx? WebsiteKey=5aa40243-c5bc-4d65-8700-ec72b9c7cb44.

13. Epstein RM: Assessment in medical education. N Engl J Med. 2007, 356:387-396.

14. Flight aware. (2019). Accessed: February 27, 2019: https://flightaware.com/.

15. Google maps. (2019). Accessed: February 27, 2019: https://www.google.com/maps/dir/St.+John's+International+Airport+ (YYT),+World+Parkway,+St.+John's,+NL/Health+Sciences....

16. Moren ME, Álamos F: Subdural hematoma bt. Critical Findings in Neuroradiology. Nunes RH, Abello AL, Castillo M (ed): Springer International Publishing, Switzerland; 2016. 225-230. 10.1007/978-3-319-27987-9

17. Moreno ME, Álamos F: Epidural hematoma. Critical Findings in Neuroradiology. Hoffmann 


\section{Cureus}

Nunes R, Abello AL, Castillo M (ed): Springer International Publishing, Switzerland; 2016. 219-224. 10.1007/978-3-319-27987-9_22 